\title{
Quantification system for the viral dynamics of a highly pathogenic simian/human immunodeficiency virus based on an in vitro experiment and a mathematical model
}

Shingo Iwami ${ }^{1,2,3,6^{*}}$, Benjamin P Holder ${ }^{4}$, Catherine AA Beauchemin ${ }^{4}$, Satoru Morita ${ }^{5}$, Tetsuko Tada ${ }^{3}$, Kei Sato ${ }^{3}$, Tatsuhiko Igarashi ${ }^{3}$ and Tomoyuki Miura ${ }^{3 *}$

\begin{abstract}
Background: Developing a quantitative understanding of viral kinetics is useful for determining the pathogenesis and transmissibility of the virus, predicting the course of disease, and evaluating the effects of antiviral therapy. The availability of data in clinical, animal, and cell culture studies, however, has been quite limited. Many studies of virus infection kinetics have been based solely on measures of total or infectious virus count. Here, we introduce a new mathematical model which tracks both infectious and total viral load, as well as the fraction of infected and uninfected cells within a cell culture, and apply it to analyze time-course data of an SHIV infection in vitro.

Results: We infected HSC-F cells with SHIV-KS661 and measured the concentration of Nef-negative (target) and Nef-positive (infected) HSC-F cells, the total viral load, and the infectious viral load daily for nine days. The experiments were repeated at four different MOls, and the model was fitted to the full dataset simultaneously. Our analysis allowed us to extract an infected cell half-life of $14.1 \mathrm{~h}$, a half-life of SHIV-KS661 infectiousness of $17.9 \mathrm{~h}, \mathrm{a}$ virus burst size of 22.1 thousand RNA copies or $0.19 \mathrm{TCID}_{50}$, and a basic reproductive number of 62.8. Furthermore, we calculated that SHIV-KS661 virus-infected cells produce at least 1 infectious virion for every 350 virions produced.
\end{abstract}

Conclusions: Our method, combining in vitro experiments and a mathematical model, provides detailed quantitative insights into the kinetics of the SHIV infection which could be used to significantly improve the understanding of SHIV and HIV-1 pathogenesis. The method could also be applied to other viral infections and used to improve the in vitro determination of the effect and efficacy of antiviral compounds.

Keywords: Viral infectiousness, Quantification of viral dynamics, In vitro experiment, Mathematical model, Simian/ Human immunodeficiency virus

\section{Background}

Historically, the study of the highly pathogenic simian/ human immunodeficiency virus (SHIV) has provided important information for the understanding of human immunodeficiency virus type-1 (HIV-1) pathogenesis. For example, it was clarified in an SHIV animal study

\footnotetext{
* Correspondence: siwami@ms.u-tokyo.ac.jp; tmiura@virus.kyoto-u.ac.jp 'Precursory Research for Embryonic Science and Technology (PRESTO), Japan Science and Technology Agency (JST), Kawaguchi, Saitama 332-0012, Japan ${ }^{3}$ Institute for Virus Research, Kyoto University, Kyoto, Kyoto 606-8507, Japan Full list of author information is available at the end of the article
}

that co-receptor usage determined by the HIV-1 env gene affects the virus' cell tropism (preference for specific target cell populations), and thus its pathogenesis, in vivo [1-3]. Furthermore, infections with highly pathogenic SHIV strains in animal models have exhibited stable clinical manifestations in most infected animals, similar to an aspect of infection course in human HIV infections $[4,5]$. One of the highly pathogenic SHIV strains, SHIV-KS661, which has the env gene of HIV-1 89.6 and predominantly uses CXCR4 as the secondary receptor for its infection [2], causes an infection that

\section{C) Biomed Central}


systemically depletes the $\mathrm{CD} 4^{+} \mathrm{T}$ cells of rhesus macaques within 4 weeks after infection [6,7]. In observations by our group in recent years, the intravenous infection of rhesus macaques with SHIV-KS661 has consistently resulted in high viremia and $\mathrm{CD}^{+}{ }^{+} \mathrm{T}$ cell depletion, followed by malignant morbidity as a result of severe chronic diarrhea and wasting after 6 to 18 months [8]. Despite this well-developed in vivo model, the detailed kinetics of SHIV-KS661 remain unclear. Quantifying and understanding viral kinetics will provide us with novel insights about the pathogenesis of SHIV (and HIV-1), for example, by enabling the quantitative comparison of the replicative capacity of different strains.

In recent years, virological data from clinical patient studies, animal experiments, and cell culture studies have frequently been analyzed using mathematical models. Mathematical analysis of clinical data is an increasingly popular tool for the evaluation of drugs, the elaboration of diagnostic criteria, and the generation of recommendations for effective therapies [9-17]. Analyses of animal and cell culture studies have revealed fundamental aspects of viral infections including the specification of the half-life of infected cells and virus, the virus burst size, and the relative contribution of the immune response [18-29]. Important results have also been obtained in the analysis of purely in vitro experiments. For example, in Beauchemin et. al. [19], simple mathematical models were employed to analyze the effect of amantadine treatment on the course of experimental infections of Madin Darby canine kidney (MDCK) cells with influenza A/Albany/1/98 (H3N2) in a hollow-fiber (HF) reactor. Fits of the models to the experimental data determined that the $50 \%$ inhibitory concentration $\left(\mathrm{IC}_{50}\right)$ of amantadine for that particular strain was 0.3 $0.4 \mu \mathrm{M}$ and found amantadine to be $56-74 \%$ effective at blocking the infection of target cells. Thus, analyses of experimental data using mathematical models have provided, and continue to provide, quantitative information about the kinetics of viral infections - particularly for HIV-1, the hepatitis $\mathrm{C}$ virus ( $\mathrm{HCV}$ ), and the influenza virus - by estimating infection parameters buried within experimental data.

Despite these successes, the available virological data, even for in vitro experiments, have often been limited in that many modeling analyses have been based only on total viral load data (e.g., RNA or DNA copies, hemagglutination assay (HA)) [9-13,15-17,20,22,23,26,27] or infectious viral load data (e.g., 50\% tissue culture infection dose $\left(\mathrm{TCID}_{50}\right)$ or plaque forming units (PFU)) $[18,19,25]$. Thus, while the applied mathematical models typically depend on the interaction of many components of the infection - including the populations of susceptible and infected cells - they are often only confronted by a single biological quantity: the time-course of the viral load. More rarely, diverse data sets including both virus and cell measurements have been considered [14,29-37]. Notable examples of the latter case include the analysis of an influenza infection in a microcarrier culture by Schulz-Horsel et al. [29], who measured and modeled the infectious and total viral load, along with the fraction of infected cells; and the in vivo studies of HIV-1 dynamics following antiviral therapy by Perelson and co-workers (e.g., $[14,31])$, who have considered measurements of viral load as well as susceptible and infected cells.

Here, we combined a relatively simple mathematical model of SHIV infection in HSC-F cells with an in vitro experimental system which allows for the measurement of both total and infectious viral load and the concentration of target and infected cells. We infected HSC-F - a $\mathrm{CD}^{+} \mathrm{T}$ cell line established from cynomolgus monkey in vitro with SHIV-KS661 at four different multiplicities of infection (MOI) and measured the concentration of Nef-negative (susceptible/target) and Nef-positive (infected/virus producing) HSC-F cells [cells/ml], and the total [RNA copies/ml] and infectious $\left[\mathrm{TCID}_{50} / \mathrm{ml}\right.$ ] viral load daily over nine days. With this abundant and diverse data, we were able to fully parameterize the dynamic model and determine robust estimates for viral kinetics parameters, thus quantifying the infection cycle. Our in vitro quantification system for SHIV-KS661 should be a valuable complement to the well-developed in vivo model and can be used to significantly improve the understanding of SHIV and HIV-1 pathogenesis.

\section{Results}

\section{Mathematical model}

To describe the in vitro kinetics of the SHIV-KS661 viral infection in our experimental system (Table 1), we expanded a basic mathematical model widely used for analyzing viral kinetics [13,17-19,27,38,39]. The following equations are our extended model:

$$
\begin{aligned}
& \frac{d x}{d t}=-\beta x v_{I}-d x \\
& \frac{d y}{d t}=\beta x v_{I}-a y \\
& \frac{d v_{I}}{d t}=p k y-r_{I} v_{I}-r_{R N A} v_{I} \\
& \frac{d v_{N I}}{d t}=(1-p) k y+r_{I} v_{I}-r_{R N A} v_{N I}
\end{aligned}
$$

where $x$ and $y$ are the number of target (susceptible) and infected (virus-producing) cells per $\mathrm{ml}$ of medium, 
Table 1 Experimental data for the in vitro experiment

\begin{tabular}{|c|c|c|c|c|c|c|c|c|c|}
\hline \multirow[t]{2}{*}{ MOI } & \multicolumn{9}{|c|}{ Measurement day } \\
\hline & 0 & 1 & 2 & 3 & 4 & 5 & 6 & 7 & 8 \\
\hline \multicolumn{10}{|c|}{ Concentration of Nef-negative HSC-F cells (cells/ml) } \\
\hline $2 \times 10^{-3}$ & 5470829 & 6044623 & 2690861 & 1012828 & 223584 & 42130 & 58470 & 10386 & 10270 \\
\hline $2 \times 10^{-4}$ & 2333804 & 4953074 & 2985268 & 2201172 & 811240 & 621750 & 60255 & 19998 & 4857 \\
\hline $2 \times 10^{-5}$ & 2574201 & 3563431 & 3434160 & 2345412 & 1269216 & 1345728 & 264794 & 71792 & 127996 \\
\hline $2 \times 10^{-6}$ & 3357117 & 2583058 & 4557411 & 35074989 & 1334060 & 1896048 & 1022157 & 307908 & 153360 \\
\hline \multicolumn{10}{|c|}{ Concentration of Nef-positive HSC-F cells (cells/ml) } \\
\hline $2 \times 10^{-3}$ & d.I..$^{*}$ & d.l. & 439139 & 1167172 & 736416 & 177870 & 41530 & 19614 & 9730 \\
\hline $2 \times 10^{-4}$ & d.l. & d.l. & 84732 & 158828 & 548760 & 878250 & 89745 & 40002 & 5143 \\
\hline $2 \times 10^{-5}$ & d.l. & d.l. & d.l. & 64588 & 170784 & 574272 & 165206 & 88208 & 92004 \\
\hline $2 \times 10^{-6}$ & d.l. & d.l. & d.l. & d.l. & 65940 & 383952 & 347843 & 232092 & 86640 \\
\hline \multicolumn{10}{|c|}{ Total viral load of SHIV-KS661 (RNA copies/ml) } \\
\hline $2 \times 10^{-3}$ & 9180000 & 331000000 & 2840000000 & 4050000000 & 3140000000 & 1120000000 & 154000000 & 20200000 & 5650000 \\
\hline $2 \times 10^{-4}$ & 1030000 & 26200000 & 256000000 & 1670000000 & 2110000000 & 1740000000 & 609000000 & 134000000 & 19400000 \\
\hline $2 \times 10^{-5}$ & 126744 & 4370000 & 51200000 & 489000000 & 1280000000 & 1940000000 & 1230000000 & 570000000 & 130000000 \\
\hline $2 \times 10^{-6}$ & 10170 & 800536 & 4600000 & 54200000 & 322000000 & 1300000000 & 1210000000 & 603000000 & 275000000 \\
\hline \multicolumn{10}{|c|}{ Infectious viral load of SHIV-KS661 (TCID $\left.{ }_{50} / \mathrm{ml}\right)$} \\
\hline $2 \times 10^{-3}$ & 40 & 4064 & 40960 & 81920 & 163840 & 20480 & 2560 & 160 & d.l. \\
\hline $2 \times 10^{-4}$ & d.l. & 101 & 403 & 5120 & 16255 & 40960 & 1280 & 101 & 40 \\
\hline $2 \times 10^{-5}$ & d.l. & 64 & 640 & 4064 & 20480 & 25803 & 5120 & 1280 & 640 \\
\hline $2 \times 10^{-6}$ & 40 & 40 & 80 & 640 & 5120 & 1280 & 1280 & 640 & 1280 \\
\hline
\end{tabular}

"d.I." designates samples in which the concentration was below the detection limit.

$v_{I}$ and $v_{N I}$ are the number of RNA copies of infectious and non-infectious virus per $\mathrm{ml}$ of medium, respectively. Parameters $d, a, r_{R N A}$, and $\beta$ represent the death rate of target cells, the death rate of infected cells, the degradation rate of viral RNA, and the rate constant for infection of target cells by virus, respectively. We assume that each infected cell releases $k$ virus particles per day (i.e., $k$ is the viral production rate of an infected cell), of which a fraction $p$ are infectious and 1-p are non-infectious. Infectious virions lose infectivity at rate $r_{I}$, becoming non-infectious. Implicit in Eqs.(1)-(4) is the assumption that once a cell is infected by infectious virus it immediately begins producing progeny virus. We also tested a variant of the model which incorporates an "eclipse" phase of infection to represent the cell's period of latency prior to virus production. We found, however, that including this phase did not significantly improve the fit of the model to the data and led to very similar extracted parameter values (see Additional files 1, 2, 3). Therefore, in all further analyses, this phase was omitted in favor of the simpler model formulation. A schematic of our mathematical model is shown in Figure 1.

To fit the observed viral load data - consisting of RNA copies $/ \mathrm{ml}$ and $\mathrm{TCID}_{50} / \mathrm{ml}$ - and to account for the partial removal of cells and virus due to sampling, we transformed Eqs.(1)-(4) into the following scaled model:

$$
\frac{d x}{d t}=-\beta_{50} x v_{50}-d x-\delta x
$$

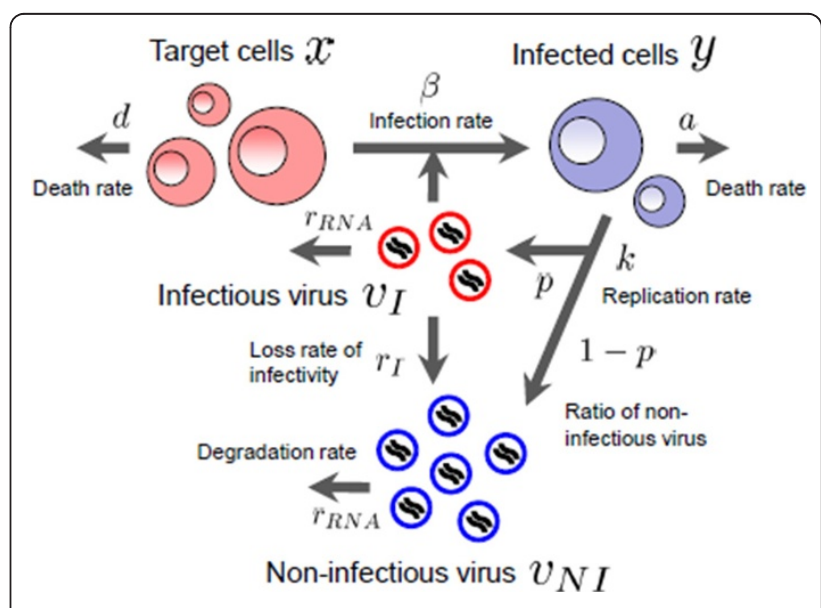

Figure $1 \mathrm{~A}$ schematic representation of the mathematical model. The variables $x$ and $y$ are the number of target and infected cells; $v_{l}$ and $v_{N_{l}}$ are the number of RNA copies of infectious and non-infectious virus, respectively. Parameters $d, a, r_{R N A}$, and $\beta$ represent the death rate of target cells, the death rate of infected cells, the degradation rate of viral RNA, and the rate constant for infection of cells by infectious virus, respectively. It is assumed that infected cells release virus particles at a rate $k$, that a fraction $p$ of these particles are infectious (1- $p$ are non-infectious) but lose infectivity at a rate $r_{l}$, becoming non-infectious. 


$$
\begin{aligned}
& \frac{d y}{d t}=\beta_{50} x v_{50}-a y-\delta y \\
& \frac{d v_{R N A}}{d t}=k y-r_{R N A} v_{R N A}-r_{c} v_{R N A} \\
& \frac{d v_{50}}{d t}=k_{50} \gamma-r_{I} v_{50}-r_{R N A} v_{50}-r_{c} v_{50}
\end{aligned}
$$

where $v_{R N A}=v_{I}+v_{N I}$ is the total concentration of viral RNA copies, $v_{50}=\alpha v_{I}$ is the infectious viral load expressed in $\mathrm{TCID}_{50} / \mathrm{ml}$, and $\alpha$ is the conversion factor from infectious viral RNA copies to $\operatorname{TCID}_{50}$. Since the measure of $1 \mathrm{TCID}_{50}$ corresponds to an average of 0.68 infection events (by Poisson statistics), we have 0 $<\alpha \leqq 1.47$ TCID $_{50}$ per RNA copies of infectious virus. Parameters $\beta_{50}=\beta / \alpha$ and $k_{50}=\alpha p k$ are the converted infection rate constant and production rate of infectious virus, respectively. At each sampling time, the concentration of Nef-negative and Nef-positive HSC-F cells must be reduced in our model by $5.5 \%$ and the viral loads (RNA copies and $\mathrm{TCID}_{50}$ ) by $99.93 \%$ to account for the experimental harvesting of cells and virus. These losses were modeled in Eqs.(5)-(8) by approximating the sampling of cells and virus as a continuous exponential decay, yielding a rate of $\delta=0.057$ per day for cell harvest and $r_{c}=7.31$ per day for virus harvest. We found that a model which implements the sampling explicitly, as a punctual reduction at each sampling time, similar to the model in [19], did not significantly improve the quality of the fit (data not shown).
Of the seven free model parameters remaining, three of them $\left(d, r_{I}, r_{R N A}\right)$ were determined by direct measurements in separate experiments described below. The remaining four parameters $\left(\beta_{50}, a, k, k_{50}\right)$ along with 16 initial $(t=0)$ values for the variables (four at each of the four MOI values) were determined by fitting the model to the data as described in Methods (Tables 2 and 3).

\section{In vitro half-lives of the SHIV-KS661 virus and HSC-F cells}

The rates at which SHIV-KS661 virions lose infectivity, $r_{I}$, and the rate at which their viral RNA degrades, $r_{R N A}$, were each estimated directly in separate experiments (Figure 2). Linear regressions were performed to fit $\log v_{R N A}(t)=\log v_{R N A}(0)-r_{R N A} t$ and $\log v_{50}(t)=\log v_{50}(0)-r_{I} t$ to those data, yielding values of $r_{R N A}=0.039$ per day (95\% confidence interval (95\%CI): $0.013-0.065$ per day) and $r_{I}=0.93$ per day (95\%CI: 0.44-1.4 per day). These correspond to an infectious virion half-life of $17.9 \mathrm{~h}$ and an RNA viability half-life of $17.7 \mathrm{~d}$. The death rate of target cells, $d$, was also estimated directly, in a mock infection experiment where Nef-negative (target) HSC-F cells were exposed to the culture conditions of the experiment without virus (data not shown). A linear regression was performed to fit $\log x(t)=\log x(0)-(d+\delta) t$ to the time course data, yielding $d=0.21$ per day $(95 \%$ CI: 0.18-0.27), corresponding to an average target cell lifespan of $4.76 \mathrm{~d}$ (half-life of $3.30 \mathrm{~d}$ ).

\section{Time-course in vitro data}

Time-course in vitro experimental data were collected

\begin{tabular}{|c|c|c|c|c|}
\hline Parameter Name & Symbol & Unit & Value & $95 \% \mathrm{Cl}$ \\
\hline \multicolumn{5}{|c|}{ Calculated parameters for the continuous approximation of cell and virus harvest } \\
\hline Harvest rate of target and infected cells & $\delta$ & day $^{-1}$ & 0.057 & - \\
\hline Harvest rate of total and infectious virus & $r_{c}$ & day $^{-1}$ & 7.31 & - \\
\hline \multicolumn{5}{|l|}{ Fitted parameters from separate experiments } \\
\hline Decay rate of uninfected cells & $d$ & day $^{-1}$ & 0.21 & $0.17-0.26$ \\
\hline Rate of virion infectivity loss & $r_{1}$ & day $^{-1}$ & 0.93 & $0.44-1.4$ \\
\hline Degradation rate of virion RNA & $r_{R N A}$ & day $^{-1}$ & 0.039 & $0.013-0.065$ \\
\hline \multicolumn{5}{|c|}{ Parameters obtained from simultaneous fit to full in vitro dataset } \\
\hline Rate constant for infections & $\beta_{50}$ & $\left(\mathrm{TCID}_{50} / \mathrm{ml} \cdot \text { day }\right)^{-1}$ & $4.95 \times 10^{-5}$ & $(2.35-9.59) \times 10^{-5}$ \\
\hline Decay rate of infected cells & a & day $^{-1}$ & 1.18 & $0.85-1.26$ \\
\hline Production rate of total virus & k & RNA copies day $^{-1}$ & $2.61 \times 10^{4}$ & $(1.55-3.70) \times 10^{4}$ \\
\hline Production rate of infectious virus & $k_{50}$ & $\mathrm{TCID}_{50} \cdot \mathrm{day}^{-1}$ & 0.22 & $0.12-0.40$ \\
\hline \multicolumn{5}{|l|}{ Quantities derived from fitted values } \\
\hline Viral burst size (total) & $k / a$ & RNA copies & $2.21 \times 10^{4}$ & $(1.74-2.96) \times 10^{4}$ \\
\hline Viral burst size (infectious) & $k_{50} d a$ & $\mathrm{TCID}_{50}$ & 0.19 & $0.11-0.33$ \\
\hline Basic reproductive number (without removal) & $R_{0}$ & - & 62.8 & $51.1-76.8$ \\
\hline Basic reproductive number (with removal) & $R_{0}^{*}$ & - & 7.01 & $5.70-8.45$ \\
\hline Minimum fraction of infectious virus & $k_{50} / k$ & $\mathrm{TCID}_{50} / \mathrm{RNA}$ copies & $8.63 \times 10^{-6}$ & $(4.53-16.9) \times 10^{-6}$ \\
\hline
\end{tabular}
over nine days, consisting of the concentrations of Nef-

Table 2 Parameters values and derived quantities for the in vitro experiment 
Table 3 Fitted initial $(t=0)$ values for the in vitro experiment

\begin{tabular}{|c|c|c|c|c|c|}
\hline \multirow[t]{2}{*}{ Variable } & \multirow[t]{2}{*}{ Unit } & \multicolumn{4}{|c|}{ Fitted initial value at $\mathrm{MOI}$ of } \\
\hline & & $2 \times 10^{-3}$ & $2 \times 10^{-4}$ & $2 \times 10^{-5}$ & $2 \times 10^{-6}$ \\
\hline$x_{j}(0)$ & cells/ml & $6.55 \times 10^{6}$ & $6.50 \times 10^{6}$ & $5.82 \times 10^{6}$ & $4.94 \times 10^{6}$ \\
\hline$y_{j}(0)$ & cells/ml & $6.47 \times 10^{2}$ & $1.60 \times 10^{2}$ & $6.89 \times 10^{-3}$ & 0.254 \\
\hline$V_{R N A j}(0)$ & RNA copies/ml & $9.15 \times 10^{6}$ & $1.05 \times 10^{6}$ & $1.58 \times 10^{5}$ & $8.21 \times 10^{3}$ \\
\hline$v_{50 j}(0)$ & $\mathrm{TCID}_{50} / \mathrm{ml}$ & 43.1 & 0.162 & 2.92 & 2.99 \\
\hline
\end{tabular}

negative and Nef-positive HSC-F cells [cells/ml], the total SHIV-KS661 viral load [RNA copies/ml], and the infectious viral load $\left[\mathrm{TCID}_{50} / \mathrm{ml}\right]$. At each daily measurement, almost all of the culture supernatant (99.93\%) was removed for viral counting; a small percentage of cells (5.5\%) were removed for counting and FACS analysis, and the remaining cells were thoroughly washed and replaced in fresh medium. The experiment was repeated for four different values of the initial viral inoculum (MOI). In total, we obtained 130 data points for quantifying SHIV-KS661 viral kinetics in vitro (Table 1 and Figure 3).

In examining the MOI $=2 \times 10^{-3}$ data, one can see that the target cell population remains high (near its initial value of approximately $6.46 \times 10^{6}$ cells $/ \mathrm{ml}$ ) until just before the peak of the virus concentration, at which point the target cell population decreases rapidly. The total infected cell population, the total virus count (RNA/ml), and the infectious virus count $\left(\mathrm{TCID}_{50} / \mathrm{ml}\right)$ all peak around $t=3$ days. Moreover, the rate of exponential decay (downward slope) of the total virus and the infected cell population after their respective peaks are quite similar. This behavior is expected: since the virus is being almost completely removed from the culture on a daily basis due to sampling and the RNA degradation rate is very small $\left(r_{R N A}=0.039\right.$ per day); the measured RNA count of virus is nearly equal to the total number of virus produced over the preceding day which should be proportional to the number of cells producing virus. Similar reasoning should apply to the decay of infectious virus - the net infectious virus measured after one day should also be approximately proportional to the number of infected cells - but the rates appear much less closely aligned in this case, perhaps due to larger errors in the TCID 50 measurement technique. Alternatively, the observed more rapid than expected decrease of infectious virus could have a biological cause. For instance, the co-infection of cells by competent and defective interfering viruses at late stages in the experiment could lead to an enhanced production of the latter [40], thus successively reducing the fraction of infectious particles. An increase in cell-death by-products could also contribute to the decline in virus infectivity. In SIV and SHIV infections in vivo, a decreasing viral infectiousness has been observed over time

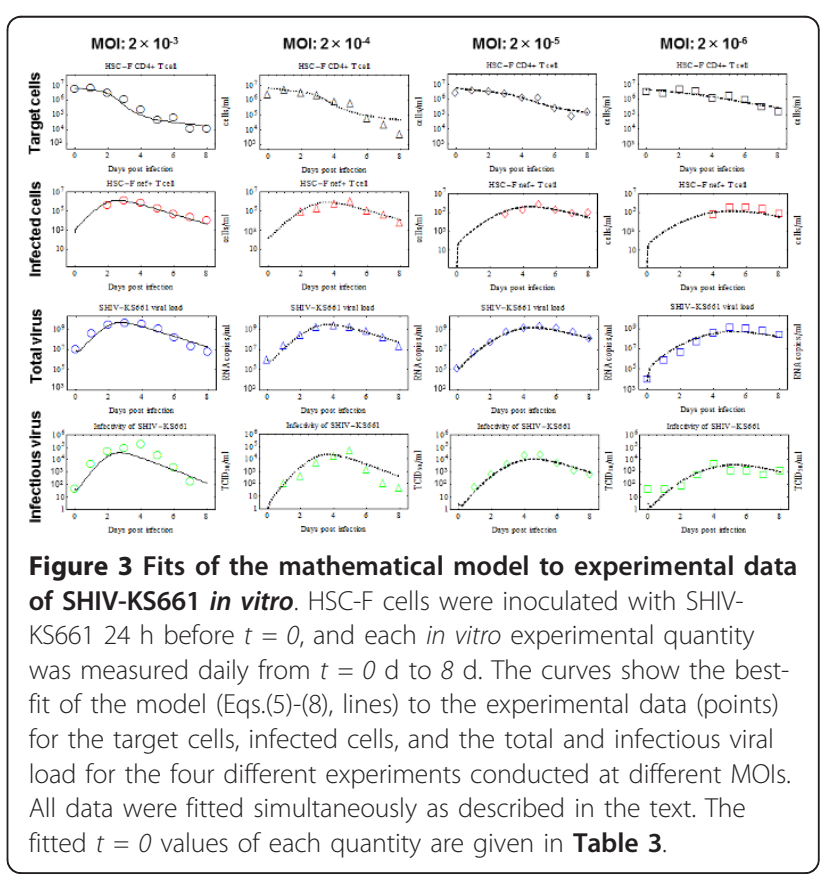


$[7,41,42]$, but the timescale of this decay is longer than that observed here and likely has an in-host origin.

A comparison of the experiments at the four different MOI values shows that a decrease in the initial viral inoculum serves primarily to delay the course of the infection. The target cell populations drop to approximately half of their original values at $t \approx 1.9,2.6,3.5$ and 4.1 days, respectively, for the four experiments in order of decreasing MOI. Similarly the peaks of the total viral RNA occur at $t \approx 3.0,4.0,5.0$ and 5.5 days, respectively. The experiments at lower MOI have slightly lower viral and infected cell peaks, but differ from those of the experiment at MOI $=2 \times 10^{-3}$ by less than a factor of three.

\section{Relevant SHIV-KS661 viral kinetics measures}

Having fixed the values of the rates of virion decay $\left(r_{I}\right.$ and $\left.r_{R N A}\right)$ and the target cell death rate $(d)$ using separate experiments, we estimated the values and $95 \% \mathrm{CI}$ of the four remaining unknown parameters $\left(\beta_{50}, a, k\right.$, $k_{50}$ ) by fitting the model in Eqs.(5)-(8) to the full in vitro dataset simultaneously (Table 2). The death rate of infected cells was determined to be $a=1.18$ per day (95\%CI: $0.85-1.26$ per day) which implies that the halflife of infected cells (i.e., $\log 2 / a$ ) is $14.1 \mathrm{~h}$. Infected HSCF cells were found to produce $k=2.61 \times 10^{4} \mathrm{RNA}$ copies of virus per day.

From the directly fitted parameters, we also calculated a number of important derived quantities and their $95 \%$ CI, determined from the bootstrap fits (Table 2). One key measurement of viral kinetics is the viral burst size, which is the total number of virus produced by an infected cell during its lifetime [18-20]. The total burst size of SHIV-KS661 (including non-infectious and infectious virus) is given in our model by $k / a$ and was estimated from our in vitro experiment to be $2.21 \times 10^{4}$ RNA copies. The burst size of infectious SHIV-KS661, $k_{50} / a$, was $0.19 \mathrm{TCID}_{50}$.

To broadly characterize viral kinetics, it is instructive to calculate the basic reproductive number for the system, which has the form $R_{0}=\beta_{50} k_{50} x_{0} /\left(a\left(r_{I}+r_{R N A}\right)\right)$ and is interpreted as the number of newly infected cells intrinsically generated by a single infectious cell at the start of the infection $[15-19,27]$. The initial number of HSC-F cells, $x_{0}$, was approximately $6.46 \times 10^{6}$ cells $/ \mathrm{ml}$, which, together with the values of the five estimated parameters, yields an estimate for the basic reproductive number of 62.8 . This large value $(62.8 » 1)$ implies that, given a small initiating infected cell population, the infection is overwhelmingly likely to spread to the entire population of cells.

After the repetitive removal of cells and virus begins, the basic reproductive number is effectively reduced, much like the effect of quarantine on the epidemiological measure of $R_{0}$. When the effects of removal are included in the calculation of the basic reproductive number it has the form $R_{0}{ }^{*}=\beta_{50} k_{50} x_{0} /((a$ $\left.+\delta)\left(r_{I}+r_{R N A}+r_{C}\right)\right)$ which yields a smaller value of 7.01. This value better characterizes the course of the infection in our system, for example, through the recursive relation for the approximate fraction of eventually infected cells, $f_{I}=1-\exp \left(-R_{O} * f_{I}\right)$ [43]. Using this expression, we find that the fraction of target cells at the end of the infection $\left(1-f_{I}\right)$ should be $9.01 \times 10^{-4}$, which implies an approximately final target cell concentration is $5.87 \times 10^{3}$ cells $/ \mathrm{ml}$. This value agrees well with the asymptotic concentration of Nef-negative HSC-F cells in the MOI $=2 \times 10^{-3}$ experiment, $\sim 1.03 \times 10^{4}$ cells $/ \mathrm{ml}$. The delay of the infection precludes an estimate of the final target cell value at smaller MOI values.

Our model formulation also enables us to determine, albiet not fully, two interesting quantities related to the infectiousness of SHIV-KS66 virions. Parameter $p$ (where $0<p \leqq 1$ ) is the fraction of SHIV-KS66 virions which are infectious at the time of production: the larger the value of $p$, the fewer defective virus particles are produced by infectious cells. Parameter $\alpha$ is approximately the fraction of infectious virions which are measured in the $\mathrm{TCID}_{50}$ assay, i.e., it is the ratio of $\mathrm{TCID}_{50}$ viral titer $\left(v_{50}\right)$ to the RNA count of infectious virions $\left(v_{I}\right)$. It follows from Poisson statistics that $0<\alpha \leqq 1.47$ $\mathrm{TCID}_{50}$ per infectious RNA copies of infectious virions. While we cannot determine $p$ and $\alpha$ individually in our analysis, their product is given by $k_{50} / k=(\alpha p k) / k=\alpha p$ $=8.63 \times 10^{-6} \mathrm{TCID}_{50}$ per infectious RNA copies. Because of the upper bounds on $p$ and $\alpha$, the value of their product imposes a minimum condition on each: $5.87 \times 10^{-6}<p \leqq 1$ and $8.63 \times 10^{-6}<\alpha \leqq 1.47 \mathrm{TCID}_{50}$ per RNA copies.

We can constrain these parameters further by considering the basic reproductive number $R_{O}=62.8$, which implies that one infectious cell will infect 62.8 other cells over the course of its infectious lifespan. Thus, one infectious cell must produce at least 62.8 infectious virions over its lifespan, i.e., have a burst size of at least 62.8 infectious RNA copies. The burst size in infectious virions is given by $p k / a$, so this requirement can be written as $p k / a \geq R_{0}$ infectious RNA copies (or, equivalently, $p \geq a R_{0} / k$ infectious RNA copies) which, based on the values of these quantities from Table 2 implies that $p \geq$ $2.84 \times 10^{-3}$. Thus $2.84 \times 10^{-3} \leqq p \leqq 1$, which means that at least one in every 350 virions produced is infectious. Since $\alpha p=8.63 \times 10^{-6}$ TCID $_{50}$ per infectious RNA copies, it follows that $8.63 \times 10^{-6}<\alpha \leqq 3.04 \times 10^{-3}$ $\mathrm{TCID}_{50}$ per infectious RNA copies, which means that 1 $\mathrm{TCID}_{50}$ corresponds to at least $330\left(1 / 3.04 \times 10^{-3}\right)$ infectious virus, but perhaps as many as $120,000(1 / 8.63 \times$ $\left.10^{-6}\right)$. 


\section{Discussion}

We have applied a simple mathematical model to quantitatively characterize the in vitro kinetics of SHIVKS661 virus infection in HSC-F cell cultures, leveraging experimental data for total and infectious viral load, along with target and infected cell dynamics, to fully parameterize the system. Specifically, we determined values for the rate of loss of infectivity and the RNA degradation rate of SHIV-KS661, the target and infected HSC-F cell half-life, the rate constant for infection of target cells and the infectious and total viral production rates of infected cells. From these fundamental quantities, we also estimated a number of important derived quantities, including the burst size of an infected cell and the basic reproductive number. Additionally, by measuring both the total and infectious viral load within the context of a mathematical model we were able to provide a lower bound for the proportion of infectious virions produced by infected cells.

We estimated the half-life of SHIV-infected HSC-F cells to be $14.1 \mathrm{~h}$. In clinical studies of patients or animals, it is extremely difficult to continuously measure the number of infected cells during infection. This is because the amount of infected cells in peripheral blood $(\mathrm{PB})$ is very small. For example, in HIV-1 infected patients, there are only about $10^{2}$ infected cells per $10^{6}$ peripheral blood mononuclear cells at their set point [14]. Thus, measuring the number of infected cells in $\mathrm{PB}$ during the early phase of infection is technically difficult. In HIV-1 humanized mice, infected cells in PB are not detectable even during the acute phase when $80-90 \%$ of target cells in the spleen and lymph nodes are infected (K. Sato and S. Iwami, unpublished data). For this reason, the death rate of infected cells in vivo has primarily been estimated from the viral load decay (or the decay of infectious virus) after the peak of an acute infection $[11,16,17,20,27]$ or after antiviral drug administration $[10,14,15,22]$. The maximum half-lives of HIV-1 and SIV-infected cells were both initially estimated - by analysis of in vivo viral decay under antiviral therapy - to be $\sim 24 \mathrm{~h}[14,27]$, but drug combinations with higher efficacy have reduced the estimates to $\sim 17$ and $\sim 11 \mathrm{~h}$, respectively $[12,22,44]$. Our in vitro estimate of the half-life, based on direct observations of Nef-positive cell decay, agrees well with these indirect in vivo measures, despite the absence of immune effects.

We determined an SHIV-KS661 viral burst size of $2.21 \times 10^{4}$ RNA or 0.19 TCID $_{50}$ for HSC-F cells. Current estimates of viral burst size in the literature rely on inhibiting multiple rounds of infection by antiviral drugs, washouts of infected cells, serial dilutions of infected cells, or infection by single-cycle virus $[11,20,21,45,46]$. The inhibition of the multiple rounds of infection, however, can introduce additional confounding factors on the viral burst size as discussed in [20]. Here, we have calculated the burst size of SHIVKS661 in HSC-F cells indirectly by estimating the viral production rate and the average lifespan of infected cells over the course of a typical infection. Our estimate is quite close to the $\sim 5 \times 10^{4}$ RNA value determined in recent SIV single-cycle virion experiments in vivo [20], which, notably, was 10-100 times higher than most previously measured values. We also calculated a basic reproductive number for SHIV-KS661 in HSC-F cell cultures as approximately 62.8 for the initial stages of the infection and approximately 7.01 for the entire course, when the effects of manual removal of virus and cells are included. The latter value implies that reducing viral growth by about $85.7 \%$ for the entire course with antiviral intervention, for example, would prevent viral spread in vitro given the daily sampling.

It is widely believed that retroviruses are predominantly defective, with less than $0.1 \%$ of virions in plasma or culture media being infectious [47-49]. On the other hand, it has recently been suggested that HIV-1 virions, for example, are inherently highly infectious, but that slow viral diffusion in liquid media and rapid dissociation of virions from cells severely limit infections in cultures (i.e., in assays measuring infectivity) [50,51]. On both sides of this debate, however, studies have often relied on measurements of the proportion of infectious virus in stock samples, or on measurements of the infectious/non-infectious ratio over the course of an in vitro experiment. These direct measurements of the infectivity ratio in a virus sample are necessarily confounded by a continuous loss of infectious virus, driven by thermal deactivation and RNA degradation and, as such, these analyses cannot address the question of what fraction of virus are infectious at the time of production. Here, we have estimated the production rates of both infectious and non-infectious virus, allowing for a novel quantitative specification of the fraction of newly generated virus that is infectious. This fundamental quantity is important in understanding the role and influence of defective virus particles [48-50,52]; and, to our knowledge, this has not been measured before for any virus strain. We determined the theoretical minimum value for the proportion of infectious virions among newly produced virus, $p$, to be $8.62 \times 10^{-6}$, by calculating the ratio of the infectious to total viral production rates $k_{50} /$ $k$. The ratio of the production rates, however, is actually $p$ multiplied by $\alpha$, where $\alpha$ is the conversion factor from RNA count of infectious virions to $\mathrm{TCID}_{50}$ (i.e., roughly the fraction of infectious virions that are actually measured in a $\mathrm{TCID}_{50}$ titration assay). Therefore, since $\alpha$ is likely much less than one, the proportion of infectious virus is likely much higher. In fact, using the 
measured basic reproductive number, we estimate that the minimum value of $p$ is approximately $2.84 \times 10^{-3}$, meaning that at least 1 of every 350 virions produced is infectious. Determining this quantity is particularly important in determining the true efficiency of infectious virus replication. In previous publications [53,54], it was reported that vif-deficient HIV-1 showed decreased production of infectious virus due to the inhibition of the viral replication process by host factors such as APOBEC3 protein. Our method suggests a novel and more reliable way to determine the effect of the host-viral protein interaction on infectious viral replication.

In another aspect of viral infectivity, we found that the SHIV-KS661 virion infectious half-life at $37^{\circ} \mathrm{C}$ was 17.9 h. While this quantity is vital for understanding viral dynamics in vitro, and represents an important, strainspecific physical property of the virion, it is unlikely to strongly influence in vivo dynamics, due to the extremely high physical clearance rate in the blood (virion half-lives are on the order of minutes) [23].

\section{Conclusions}

To conclude, by using a simple mathematical model for SHIV-KS661 infection on HSC-F cells and an abundant, diverse experimental dataset, we have been able to reliably estimate the parameters characterizing cell-virus interactions in vitro. Based on these estimated parameters, we have provided a quantitative description of SHIV-KS661 kinetics in HSC-F cell cultures which is consistent with previous studies of lentiviruses and provides a number of novel quantities. Most notably, our analysis provides an estimate of the minimum fraction of infectious virus produced by an infected cell. Our improved method for quantifying viral kinetics in vitro which depends crucially on detailed time-course information about the infection of cells in addition to that of virus (both total particle count and infectious titer) could be applied to other viral infections. The method could likely improve the understanding of the differences in replication across different strains [25,55] or between complete and protein-deficient viruses [53,54]; the differences in viral pathogenesis [6]; and the effects of anti-viral therapies [9,13]. Quantifying the in vitro viral kinetics for viruses such as HCV [56,57], for which a convenient animal experimental model has not been established, is of particular interest. Since the method presented here allows for the complete resolution of all viral kinetic parameters, it also enables the identification of the mechanisms of action for new antiviral compounds. Indeed, repeating the experimental infection under various antiviral concentrations would distinctly reveal which parameters (e.g., half-life of infected cells, infectious viral burst size) are affected by the antiviral and to what extent. Furthermore, the inhibitory concentration of the compound could be independently determined for each parameter. Thus, our synergistic approach, combining experiments and mathematical models, has broad potential applications in virology.

\section{Methods}

\section{Virus and cell culture}

The virus stock of SHIV-KS661 [5] was prepared in a $\mathrm{CD}^{+}$human $\mathrm{T}$ lymphoid cell line, M8166 (a subclone of C8166) [58]. The stock was stored in liquid nitrogen until use. Establishment of the HSC-F cell line has been previously described [59]. This is a cynomolgous monkey $\mathrm{CD}^{+}{ }^{+} \mathrm{T}$-cell line from fetal splenocytes that were immortalized by infection with Herpesvirus saimiri subtype C. The cells were cultured in RPMI-1640 medium supplemented with $10 \%$ fetal calf serum at $37^{\circ} \mathrm{C}$ and $5 \%$ $\mathrm{CO}_{2}$ in humidified condition.

\section{In vitro experiment}

Each experiment was performed using 2 wells of a 24well plate with a total suspension volume of $2 \mathrm{ml}(1 \mathrm{ml}$ per well) and an initial cell concentration of $6.46 \times 10^{6}$ cells $/ \mathrm{ml}$ in each well. Because the initial cell concentration is close to the carrying capacity of 24-well plates and the doubling time of HSC-F cells is not short, the population of target cells, in the absence of SHIV-KS661 infection, changes very little on the timescale of our experiment. We therefore neglected the effects of potential regeneration of HSC-F cells when constructing the mathematical model.

Cultures of HSC-F cells were inoculated at different MOIs $\left(2.0 \times 10^{-3}, 2.0 \times 10^{-4}, 2.0 \times 10^{-5}, 2.0 \times 10^{-6}\right.$; MOI $=$ TCID50/cell) of SHIV-KS661 and incubated for $4 \mathrm{~h}$ at $37^{\circ} \mathrm{C}$. After inoculation, cells were washed three times to remove the infection medium and placed in fresh media. Subsequently, the culture supernatant was harvested daily for $9 \mathrm{~d}$, along with a small fraction of the cells $(5.5 \%)$ for counting of viable and infected cells. The remaining cells were then gently washed three times and placed in a fresh, virus-free, medium. Separate experiments (not shown) determined that free virus was not completely removed, but that virus concentration in the supernatant dropped to $0.066 \%$ of its value prior to this sampling and washing procedure. Harvested culture supernatants were frozen and stored at $-80^{\circ} \mathrm{C}$ until they were assayed via RT-PCR and TCID $_{50}$ titration, as described below.

\section{Count of viable and infected cells}

Virus infection of the HSC-F cells was measured by FACS analysis using markers for intracellular SIV Nef antigen expression. The counts of total and viable cells were first determined using a cell counting chamber 
(Burker-turk, Erma, Tokyo, Japan) with trypan blue staining. Viable HSC-F cells (gated by forward- and side-scatter results) were examined by flow cytometry to measure the intracellular SIV Nef antigen expression (see Figure 4). Cells were permeabilized with detergentcontaining buffer (Permeabilizing Solution 2, BD Biosciences, San Jose, CA). The permeabilized cells were stained with anti-SIV Nef monoclonal antibody (04-001, Santa Cruz Biotechnology, Santa Cruz, CA) labeled by Zenon Alexa Fluor 488 (Invitrogen, Carlsbad, CA), and analyzed on FACSCalibur (BD Biosciences, San Jose, CA).

\section{Total and infectious viral load quantification}

We followed the kinetics of both the total and infectious SHIV-KS661 viral load. The total viral load was measured with a real-time PCR quantification assay, as described previously [5], with minor modifications. Briefly, total RNA was isolated from the culture supernatants $(140 \mu \mathrm{l})$ of virus-infected HSC-F cells with a QIAamp Viral RNA Mini kit (QIAGEN, Hilden, Germany). RT reactions and PCR were performed by a QuantiTect probe RT-PCR Kit (QIAGEN, Hilden, Germany) using the following primers for the gag region; SIV2-696F (5'-GGA AAT TAC CCA GTA CAA CAA ATAGG-3') and SIV2-784R (5'-TCT ATC AAT TTT ACC CAGGCA TTT A-3'). A labeled probe, SIV2-731T (5'-Fam-TGTCCA CCT GCC ATT AAG CCC GTamra-3'), was used for detection of the PCR products. These reactions were performed with a Prism 7500 Sequence Detector (Applied Biosystems, Foster City,

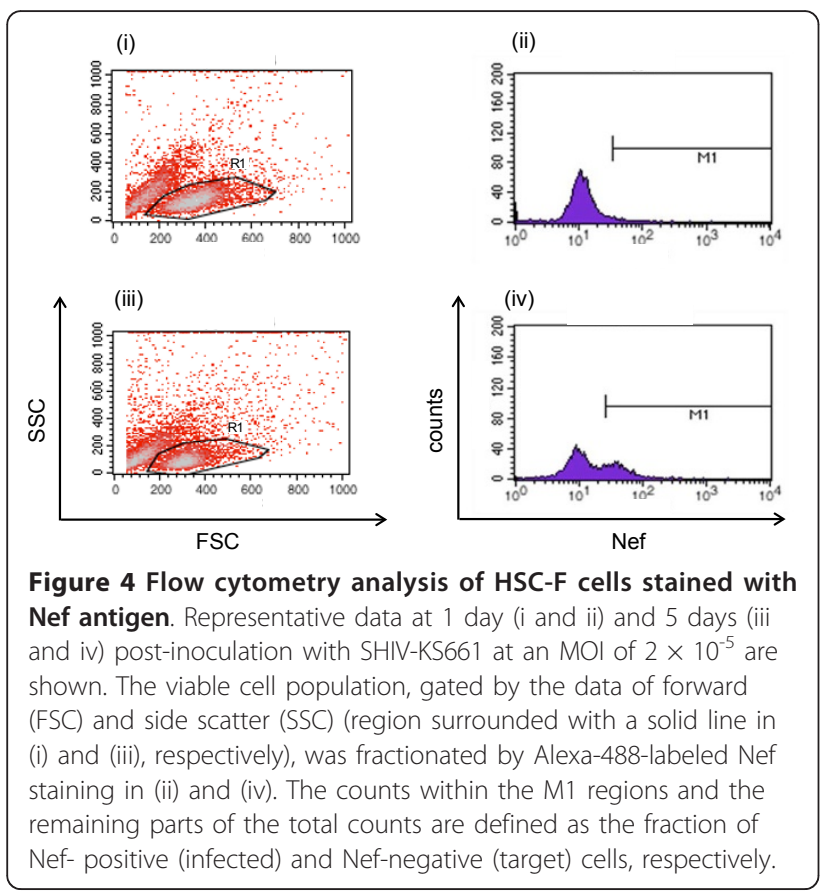

CA) and analyzed using the manufacturer's software. For each run, a standard curve was generated from dilutions whose copy numbers were known, and the RNA in the culture supernatant samples was quantified based on the standard curve. The infectious viral load was measured by TCID $_{50}$ assay in HFC-S cell cultures using 96-well flat bottom plates at cell concentrations of $1.0 \times$ $10^{6}$ cells $/ \mathrm{ml}$. The titer of the virus was determined as described by Reed and Muench [60].

\section{Rate of RNA degradation and loss of infectivity for SHIV- KS661 in the culture condition}

The RNA degradation and thermal deactivation of SHIV-KS661 was measured by incubating $4 \mathrm{ml}$ of stock virus, without cells, in a $35 \mathrm{~mm}$ Petri dish under the same conditions as the infection experiments (in RPMI1640 medium supplemented with $10 \%$ fetal calf serum at $37^{\circ} \mathrm{C}$ and $5 \% \mathrm{CO}_{2}$ in humidified condition). Aliquots of the stock $(500 \mu \mathrm{l})$ were sampled every day from day 0 to day 5 and stored at $-80^{\circ} \mathrm{C}$ (see Figure 2). The RNA copy number and $50 \%$ tissue culture infectious dose of the samples were measured as described above.

\section{Mathematical model and fitting}

We simultaneously fit Eqs.(5)-(8) to the concentration of Nef-negative and Nef-positive HSC-F cells and the infectious and total viral loads at four different MOIs (Figure 3) using nonlinear least-squares regression (FindMinimum package of Mathematica7.0) which minimizes the following objective function:

$$
\begin{aligned}
& \operatorname{SSR}=\sum_{j=1}^{4}\left[\sum_{i=1}^{9}\left\{\log x_{j}\left(t_{i}\right)-\log x_{j}^{e}\left(t_{i}\right)\right\}^{2}+\sum_{i=1}^{9}\left\{\log y_{j}\left(t_{i}\right)-\log y_{j}^{e}\left(t_{i}\right)\right\}^{2}\right. \\
& \left.+\sum_{i=1}^{9}\left\{\log v_{R N A j}\left(t_{i}\right)-\log v_{R N A j}\left(t_{i}\right)\right\}^{2}+\sum_{i=1}^{9}\left\{\log v_{50 j}\left(t_{i}\right)-\log v_{50 j}{ }^{e}\left(t_{i}\right)\right\}^{2}\right]
\end{aligned}
$$

where $x_{j}\left(t_{i}\right), y_{j}\left(t_{i}\right), v_{R N A j}\left(t_{i}\right)$, and $v_{50 j}\left(t_{i}\right)$ are the modelpredicted values for Nef-negative cells, Nef-positive cells, total RNA viral load and infectious $\left(\mathrm{TCID}_{50}\right.$ ) viral load, given by the solution of Eqs.(5)-(8) at measurement time $t_{i}\left(t_{i}=0,1,2, \ldots, 8 \mathrm{~d}\right)$. Index $j$ is a label for the MOI of the four experiments (i.e., for MOI: $2.0 \times$ $10^{-3}, 2.0 \times 10^{-4}, 2.0 \times 10^{-5}$, and $\left.2.0 \times 10^{-6}\right)$. The variables with superscript " $e$ " are the corresponding experimental measurements of those quantities. Note that the HSC-F cells were inoculated with SHIV-KS661 $24 \mathrm{~h}$ before $t=$ 0 . Experimental measurements below the detection limit (marked "d.l." in Table 1) were excluded when computing the SSR. Alternative fits with various weights on the infectious viral load to account for larger errors in the $\mathrm{TCID}_{50}$ value [61], were also performed, but these did not significantly alter the extracted parameter values (Additional files 4, 5, 6, 7, 8, 9). To derive the 95\% confidence interval for each parameter, we employed the 
bootstrap method [62,63], estimating parameter values using 256 replicates of the four data sets and calculating the 2.5 and 97.5 percentiles.

\section{Additional material}

\section{Additional file 1: Fit of a mathematical model which includes an eclipse phase of infection to experimental data of SHIV-KS661 in vitro. Testing a variant of the model which incorporates an "eclipse" phase of infection to represent the cell's period of latency prior to virus production (see Additional file 2 for more detailed information).}

Additional file 2: Additional documentation for Additional files 1 . Detailed explanation of mathematical models used in Additional files 1.

Additional file 3: Table for estimated parameters in Additional files 1. Parameters values, initial values and derived quantities for the in vitro experiment with eclipse model.

Additional file 4: Fit of the mathematical model with $\mathrm{SSR}^{\mathrm{W}}(W=$ 0.0001 ) to experimental data of SHIV-KS661 in vitro (a). Fitting with weight of $W=0.0001$ on the infectious viral load to account for larger errors in the $T C I D_{50}$ value (see Additional file 8 for more detailed information).

Additional file 5: Fit of the mathematical model with $\mathrm{SSR}^{\mathrm{W}}(W=$ $0.1)$ to experimental data of SHIV-KS661 in vitro (b). Fitting with weight of $W=0.1$ on the infectious viral load to account for larger errors in the $\mathrm{TCID}_{50}$ value (see Additional file 8 for more detailed information).

Additional file 6: Fit of the mathematical model with $\operatorname{SSR}^{W}(W=10)$ to experimental data of SHIV-KS661 in vitro (c). Fitting with weight of $W=10$ on the infectious viral load to account for larger errors in the $\mathrm{TCID}_{50}$ value (see Additional file 8 for more detailed information).

Additional file 7: Fit of the mathematical model with $\mathrm{SSR}^{\mathrm{W}}(W=$ 10000) to experimental data of SHIV-KS661 in vitro (d). Fitting with weight of $W=10000$ on the infectious viral load to account for larger errors in the TCID 50 value (see Additional file 8 for more detailed information).

Additional file 8: Additional documentation for Additional files 4, 5, 6,7 . Detailed explanation of mathematical models used in Additional files 4, 5, 6, 7 .

Additional file 9: Table for estimated parameters in Additional files $4,5,6,7$. Parameters values and derived quantities for the in vitro experiment with various $S_{S R} \mathrm{~W}_{S}$.

\section{List of abbreviations}

SHIV: simian/human immunodeficiency virus; HIV-1: human immunodeficiency virus type-1; MDCK: Madin Darby canine kidney; HF: hollow-fiber; $\mathrm{I}_{50}$ : 50\% inhibitory concentration; HCV: hepatitis $\mathrm{C}$ virus; HA: hemagglutination assay; $\mathrm{TCID}_{50}: 50 \%$ tissue culture infection dose; PFU: plaque forming units; MOI: multiplicities of infection; PB: peripheral blood.

\section{Acknowledgements}

This work was supported, in part, by JST PRESTO program (SI) and by the Natural Sciences and Engineering Research Council of Canada (CAAB) and by a grant-in-aid for scientific research from the Ministry of Education and Science, Japan, Research on Human Immunodeficiency Virus/AIDS in Health and Labor Sciences research grants from the Ministry of Health, Labor and Welfare, Japan, a research grant for health sciences focusing on drug innovation for AIDS from the Japan Health Sciences Foundation (TM).

\section{Author details}

'Precursory Research for Embryonic Science and Technology (PRESTO), Japan Science and Technology Agency (JST), Kawaguchi, Saitama 332-0012, Japan.

${ }^{2}$ Graduate School of Mathematical Sciences, The University of Tokyo, Meguro-ku, Tokyo 153-8914, Japan. ${ }^{3}$ Institute for Virus Research, Kyoto University, Kyoto, Kyoto 606-8507, Japan. ${ }^{4}$ Department of Physics, Ryerson University, ON, Toronto M5B 2K3, Canada. ${ }^{5}$ Department of Systems
Engineering, Shizuoka University, Hamamatsu, Shizuoka 432-8561, Japan. ${ }^{6}$ Department of Biology, Faculty of Sciences, Kyushu University, 6-10-1 Hakozaki, Higashi-ku, Fukuoka, Fukuoka 812-8581, Japan.

\section{Authors' contributions}

$\mathrm{SI}, \mathrm{KS}, \mathrm{TI}$ and TM designed the study.

$\mathrm{SI}, \mathrm{BPH}$ and SM carried out data analysis.

$\Pi T$ and TM performed all experiments.

$\mathrm{SI}$ and $\mathrm{CAAB}$ developed mathematical model.

$\mathrm{SI}, \mathrm{BPH}, \mathrm{CAAB}$ and TM wrote the final manuscript.

All authors read and approved the final manuscript.

\section{Competing interests}

The authors declare that they have no competing interests.

Received: 7 October 2011 Accepted: 25 February 2012

Published: 25 February 2012

\section{References}

1. Harouse JM, Gettie A, Tan RC, Blanchard J, Cheng-Mayer C: Distinct pathogenic sequela in rhesus macaques infected with CCR5 or CXCR4 utilizing SHIVs. Science 1999, 284:816-819.

2. Matsuda K, Inaba K, Fukazawa $Y$, Matsuyama M, Ibuki K, Horiike M, Saito N Hayami M, Igarashi T, Miura T: In vivo analysis of a new R5 tropic SHIV generated from the highly pathogenic SHIV-KS661, a derivative of SHIV89.6. Virology 2010, 399:134-143.

3. Nishimura Y, Igarashi T, Donau OK, Buckler-White A, Buckler C, Lafont BA, Goeken RM, Goldstein S, Hirsch VM, Martin MA: Highly pathogenic SHIVs and SIVs target different $\mathrm{CD}^{+} \mathrm{T}$ cell subsets in rhesus monkeys explaining their divergent clinical courses. Proc Natl Acad Sci USA 2004, 101:12324-12329.

4. Reimann KA, Li JT, Veazey R, Halloran M, Park IW, Karlsson GB, Sodroski J, Letvin NL: A chimeric simian/human immunodeficiency virus expressing a primary patient human immunodeficiency virus type 1 isolate env causes an AIDS-like disease after in vivo passage in rhesus monkeys. $J$ Virol 1996, 70:6922-6928.

5. Shinohara K, Sakai K, Ando S, Ami Y, Yoshino N, Takahashi E, Someya K, Suzaki Y, Nakasone T, Sasaki Y, Kaizu M, Lu Y, Honda M: A highly pathogenic simian/human immunodeficiency virus with genetic changes in cynomolgus monkey. J Gen Virol 1999, 80:1231-1240.

6. Kozyrev IL, Ibuki K, Shimada T, Kuwata T, Takemura T, Hayami M, Miura T: Characterization of less pathogenic infectious molecular clones derived from acute-pathogenic SHIV-89.6p stock virus. Virology 2001, 282:6-13.

7. Miyake A, Ibuki K, Enose Y, Suzuki H, Horiuchi R, Motohara M, Saito N, Nakasone T, Honda M, Watanabe T, Miura T, Hayami M: Rapid dissemination of a pathogenic simian/human immunodeficiency virus to systemic organs and active replication in lymphoid tissues following intrarectal infection. J Gen Virol 2006, 13:11-20.

8. Inaba K, Fukazawa Y, Matsuda K, Himeno A, Matsuyama M, Ibuki K, Miura Y, Koyanagi Y, Nakajima A, Blumberg RS, Takahashi H, Hayami M, Igarashi T, Miura T: Small intestine $\mathrm{CD4}^{+}$cell reduction and enteropathy in simian/ human immunodeficiency virus KS661-infected rhesus macaques in the presence of low viral load. J Gen Virol 2010, 91:773-781.

9. Dixit NM, Layden-Almer JE, Layden TJ, Perelson AS: Modelling how ribavirin improves interferon response rates in hepatitis $C$ virus infection. Nature 2004, 432:922-924.

10. Ho DD, Neumann AU, Perelson AS, Chen W, Leonard JM, Markowitz M: Rapid turnover of plasma virions and CD4 lymphocytes in HIV-1 infection. Nature 1995, 373:123-126.

11. Little SJ, McLean AR, Spina CA, Richman DD, Havlir DV: Viral dynamics of acute HIV-1 infection. J Exp Med 1999, 190:841-850.

12. Markowitz M, Louie M, Hurley A, Sun E, Di Mascio M, Perelson AS, Ho DD: A novel antiviral intervention results in more accurate assessment of human immunodeficiency virus type 1 replication dynamics and T-cell decay in vivo. J Virol 2003, 77:5037-5038.

13. Neumann AU, Lam NP, Dahari H, Gretch DR, Wiley TE, Layden TJ, Perelson AS: Hepatitis C viral dynamics in vivo and the antiviral efficacy of interferon-alpha therapy. Science 1998, 282:103-107.

14. Perelson AS, Essunger $P$, Cao $Y$, Vesanen M, Hurley A, Saksela $K$, Markowitz M, Ho DD: Decay characteristics of HIV-1-infected compartments during combination therapy. Nature 1997, 387:188-191. 
15. Perelson AS, Neumann AU, Markowitz M, Leonard JM, Ho DD: HIV-1 dynamics in vivo: virion clearance rate, infected cell life-span, and viral generation time. Science 1996, 271:1582-1586.

16. Ribeiro RM, Qin L, Chavez LL, Li D, Self SG, Perelson AS: Estimation of the initial viral growth rate and basic reproductive number during acute HIV-1 infection. J Virol 2010, 84:6096-6102.

17. Stafford MA, Corey L, Cao Y, Daar ES, Ho DD, Perelson AS: Modeling plasma virus concentration during primary HIV infection. J Theor Biol 2000, 203:285-301.

18. Baccam P, Beauchemin C, Macken CA, Hayden FG, Perelson AS: Kinetics of influenza A virus infection in humans. J Virol 2006, 80:7590-7599.

19. Beauchemin CA, McSharry JJ, Drusano GL, Nguyen JT, Went GT, Ribeiro RM, Perelson AS: Modeling amantadine treatment of influenza A virus in vitro. J Theor Biol 2008, 254:439-451.

20. Chen HY, Di Mascio M, Perelson AS, Ho DD, Zhang L: Determination of virus burst size in vivo using a single-cycle SIV in rhesus macaques. Proc Natl Acad Sci USA 2007, 104:19079-19084.

21. Dimitrov DS, Willey RL, Sato $H$, Chang $L$, Blumenthal $R$, Martin MA: Quantitation of human immunodeficiency virus type 1 infection kinetics. J Virol 1993, 67:2182-2190.

22. Dinoso JB, Rabi SA, Blankson JN, Gama L, Mankowski JL, Siliciano RF, Zink MC, Clements JE: A simian immunodeficiency virus-infected macaque model to study viral reservoirs that persist during highly active antiretroviral therapy. J Virol 2009, 83:9247-9257.

23. Igarashi T, Brown C, Azadegan A, Haigwood N, Dimitrov D, Martin MA Shibata R: Human immunodeficiency virus type 1 neutralizing antibodies accelerate clearance of cell-free virions from blood plasma. Nat Med 1999, 5:211-216.

24. Miao H, Hollenbaugh JA, Zand MS, Holden-Wiltse J, Mosmann TR, Perelson AS, Wu H, Topham DJ: Quantifying the early immune response and adaptive immune response kinetics in mice infected with influenza A virus. J Virol 2010, 84:6687-6698.

25. Mitchell H, Levin D, Forrest S, Beauchemin CA, Tipper J, Knight J, Donart N, Layton RC, Pyles J, Gao P, Harrod KS, Perelson AS, Koster F: Higher level of replication efficiency of 2009 (H1N1) pandemic influenza virus than those of seasonal and avian strains: kinetics from epithelial cell culture and computational modeling. J Virol 2011, 85:1125-1135.

26. Mohler L, Flockerzi D, Sann H, Reichl U: Mathematical model of influenza A virus production in large-scale microcarrier culture. Biotechnol Bioeng 2005, 90:46-58.

27. Nowak MA, Lloyd AL, Vasquez GM, Wiltrout TA, Wahl LM, Bischofberger N, Williams J, Kinter A, Fauci AS, Hirsch VM, Lifson JD: Viral dynamics of primary viremia and antiretroviral therapy in simian immunodeficiency virus infection. J Virol 1997, 71:7518-7525.

28. Regoes RR, Barber DL, Ahmed R, Antia R: Estimation of the rate of killing by cytotoxic T lymphocytes in vivo. Proc Natl Acad Sci USA 2007 104:1599-1603.

29. Schulze-Horsel J, Schulze M, Agalaridis G, Genzel Y, Reichl U: Infection dynamics and virus-induced apoptosis in cell culture-based influenza vaccine production - Flow cytometry and mathematical modeling. Vaccine 2009, 27:2712-2722

30. Funk GA, Fischer M, Joos B, Opravil M, Gunthard H, Huldrych F, Ledergerber B, Bonhoeffer S: Quantification of In Vivo Replicative Capacity of HIV-1 in Different Compartments of Infected Cells. JAIDS 2001, 26:397-404.

31. Hlavacek WS, Stilianakis NI, Notermans DW, Danner SA, Perelson AS: Influence of follicular dendritic cells on decay of HIV during antiretroviral therapy. Proc Natl Acad Sci USA 2000, 97:10966-10971.

32. Kepler GM, Nguyen HK, Webster-Cyriaque J, Banks HT: A dynamic model for induced reactivation of latent virus. J Theor Biol 2007, 244:451-462.

33. Murray JM, Purcell RH, Wieland SF: The half-life of hepatitis B virions. Hepatology 2006, 44:1117-1121.

34. Regoes RR, Antia R, Garber DA, Silvestri G, Feinberg MB, Staprans SI: Roles of target cells and virus-specific cellular immunity in primary simian immunodeficiency virus infection. J Virol 2004, 78:4866-4875.

35. Speirs C, van Nimwegen E, Bolton D, Zavolan M, Duvall M, Angleman S, Siegel R, Perelson AS, Lenardo MJ: Analysis of human immunodeficiency virus cytopathicity by using a new method for quantitating viral dynamics in cell culture. J Virol 2005, 79:4025-4032.
36. Stilianakis $\mathrm{NI}$, Boucher $\mathrm{CAB}$, deJong MD, van Leeuwen $\mathrm{R}$, Schuurman $\mathrm{R}$, de Boer RJ: Clinical data sets of HIV1 Reverse transcriptase-resistant mutants explained by a mathematical model. J Virol 1997, 71:161-168.

37. Verotta D, Schaedeli F: Non-linear dynamics modes characterizing longterm virological data from AIDS clinical trials. Math Biosci 2002, 176:163-183

38. Nowak MA, May RM: Virus dynamics. Oxford University Press; 2000.

39. Perelson AS: Modeling viral and immune system dynamics. Nat Rev Immunol 2001, 2:28-36.

40. Bangham CRM, Kirkwood TBL: Defective interfering particles: Effects in modulating virus growth and persistence. Virology 1990, 179:821-826.

41. Ma ZM, Stone M, Piatak M, Schweighardt B, Haigwood NL, Montefiori D, Lifson JD, Busch MP, Miller CJ: High specific infectivity of plasma virus from the pre-ramp-up and ramp-up stages of acute Simian Immunodeficiency Virus infection. J Virol 2009, 83:3288-3297.

42. Vaidya NK, Ribeiro RM, Miller CJ, Perelson AS: Viral dynamics during primary Simian Immunodeficiency Virus Infection: Effect of Timedependent Virus Infectivity. J Virol 2010, 84:4302-4310.

43. Anderson RM: The Kermack-McKendrick Epidemic Threshold Theorem. Bull Math Biol 1991, 53:3-32.

44. Brandin E, Thorstensson R, Bonhoeffer S, Albert J: Rapid viral decay in simian immunodeficiency virus-infected macaques receiving quadruple antiretroviral therapy. J Virol 2006, 80:9861-9864.

45. Eckstein DA, Penn ML, Korin YD, Scripture-Adams DD, Zack JA, Kreisberg JF, Roederer M, Sherman MP, Chin PS, Goldsmith MA: HIV-1 actively replicates in naive $\mathrm{CD} 4(+) \mathrm{T}$ cells residing within human lymphoid tissues. Immunity 2001, 15:671-682.

46. Tsai WP, Conley SR, Kung HF, Garrity RR, Nara PL: Preliminary in vitro growth cycle and transmission studies of HIV-1 in an autologous primary cell assay of blood-derived macrophages and peripheral blood mononuclear cells. Virology 1996, 226:205-216.

47. Huang AS, Baltimore D: Defective viral particles and viral disease processes. Nature 1970, 226:325-327.

48. Kwon YJ, Hung G, Anderson WF, Peng CA, Yu H: Determination of infectious retrovirus concentration from colony-forming assay with quantitative analysis. J Virol 2003, 77:5712-5720.

49. Rusert P, Fischer M, Joos B, Leemann C, Kuster H, Flepp M, Bonhoeffer S, Gunthard HF, Trkola A: Quantification of infectious HIV-1 plasma viral load using a boosted in vitro infection protocol. Virology 2004, 326:113-129.

50. Platt EJ, Kozak SL, Durnin JP, Hope TJ, Kabat D: Rapid dissociation of HIV-1 from cultured cells severely limits infectivity assays, causes the inactivation ascribed to entry inhibitors, and masks the inherently high level of infectivity of virions. J Virol 2010, 84:3106-3110.

51. Thomas JA, Ott DE, Gorelick RJ: Efficiency of Human Immunodeficiency Virus Type 1 Postentry Infection Processes: Evidence against Disproportionate Numbers of Defective Virions. J Virol 2007, 81:4367-4370.

52. Marcus PI, Ngunjiri JM, Sekellick MJ: Dynamics of biologically active subpopulations of influenza virus: Plaque-forming, noninfectious cellkilling, and defective interfering particles. J Virol 2009, 83:8122-8130.

53. Izumi T, lo K, Matsui M, Shirakawa K, Shinohara M, Nagai Y, Kawahara M, Kobayashi M, Kondoh $\mathrm{H}$, Misawa N, Koyanagi Y, Uchiyama T, TakaoriKondo A: HIV-1 viral infectivity factor interacts with TP53 to induce G2 cell cycle arrest and positively regulate viral replication. Proc Natl Acad Sci USA 2010, 107:20798-20803.

54. Sato K, Izumi T, Misawa N, Kobayashi T, Yamashita Y, Ohmichi M, Ito M, Takaori-Kondo A, Koyanagi Y: Remarkable lethal G-to-A mutations in vifproficient HIV-1 provirus by individual APOBEC3 proteins in humanized mice. J Virol 2010, 84:9546-9556.

55. De Jong MD, Simmons CP, Thanh TT, Hien VM, Smith GJD, Chau TNB, Hoang DM, Chau NW, Khanh TH, Dong VC, Qui PT, Cam BV, Ha DQ, Guan Y, Peiris JSM, Chinh NT, Hien T, Farrar J: Fatal outcome of human influenza A (H5N1) is associated with high viral load and hypercytokinemia. Nat Med 2006, 12:1203-1207.

56. Wakita T, Pietschmann T, Kato T, Date T, Miyamoto M, Zhao Z, Murthy K, Habermann A, Kräusslich HG, Mizokami M, Bartenschlager R, Liang TJ: Production of infectious hepatitis $C$ virus in tissue culture from a cloned viral genome. Nat Med 2005, 11:791-796.

57. Watashi $K$, Ishii N, Hijikata M, Inoue D, Murata T, Miyanari $Y$, Shimotohno K: Cyclophilin $B$ is a functional regulator of hepatitis $C$ virus RNA polymerase. Mol Cell 2005, 19:111-122. 
58. Clapham PR, Weiss RA, Dalgleish AG, Exley M, Whitby D, Hogg N: Human immunodeficiency virus infection of monocytic and T-lymphocytic cells: receptor modulation and differentiation induced by phorbol ester. Virology 1987, 158:44-51.

59. Akari H, Mori K, Terao K, Otani I, Fukasawa M, Mukai R, Yoshikawa Y: In vitro immortalization of Old World monkey T lymphocytes with Herpesvirus saimiri: its susceptibility to infection with simian immunodeficiency viruses. Virology 1996, 218:382-388.

60. Reed $\sqcup$, Muench $\mathrm{H}$ : A simple method of estimating fifty per cent endpoints. Am J Hyg 1938, 27:493-497.

61. Macken C: Design and analysis of serial limiting dilution assays with small sample sizes. J Immunol Methods 1999, 222:13-29.

62. Efron B: Bootstrap Method: Another Look at the Jackknife. Annals of Statistic 1979, 7:1-26.

63. Efron B, Tibshirani R: Bootstrap methods for standard errors, confidence intervals, and other measures of statistical accuracy. Stat Sci 1986, $1: 54-75$

doi:10.1186/PREACCEPT-8502330256154242

Cite this article as: Iwami et al:: Quantification system for the viral dynamics of a highly pathogenic simian/human immunodeficiency virus based on an in vitro experiment and a mathematical model. Retrovirology 2012 9:18.

\section{Submit your next manuscript to BioMed Central} and take full advantage of:

- Convenient online submission

- Thorough peer review

- No space constraints or color figure charges

- Immediate publication on acceptance

- Inclusion in PubMed, CAS, Scopus and Google Scholar

- Research which is freely available for redistribution

Submit your manuscript at www.biomedcentral.com/submit 